\title{
Evolutionary origin and genomic organisation of runt-domain containing genes in arthropods Elizabeth J Duncan ${ }^{1}$, Megan J Wilson ${ }^{1}$, James M Smith ${ }^{1}$ and Peter K Dearden*1,2
}

\author{
Address: ${ }^{1}$ Laboratory for Evolution and Development, Department of Biochemistry, University of Otago, Aotearoa-New Zealand and ${ }^{2}$ National \\ Research Centre for Growth and Development, Department of Biochemistry, University of Otago, PO Box 56, Dunedin, Aotearoa-New Zealand \\ Email: Elizabeth J Duncan - elizabeth.duncan@otago.ac.nz; Megan J Wilson - meganj.wilson@stonebow.otago.ac.nz; \\ James M Smith - james.smith@otago.ac.nz; Peter K Dearden* - peter.dearden@stonebow.otago.ac.nz \\ * Corresponding author
}

Published: 25 November 2008

BMC Genomics 2008, 9:558 doi:10.1/86/147|-2164-9-558

This article is available from: http://www.biomedcentral.com/I47/-2/64/9/558

(c) 2008 Duncan et al; licensee BioMed Central Ltd.

This is an Open Access article distributed under the terms of the Creative Commons Attribution License (http://creativecommons.org/licenses/by/2.0), which permits unrestricted use, distribution, and reproduction in any medium, provided the original work is properly cited.

\begin{abstract}
Background: Gene clusters, such as the Hox gene cluster, are known to have critical roles in development. In eukaryotes gene clusters arise primarily by tandem gene duplication and divergence. Genes within a cluster are often coregulated, providing selective pressure to maintain the genome organisation, and this co-regulation can result in temporal or spatial co-linearity of gene expression. It has been previously noted that in Drosophila melanogaster, three of the four runt-domain (RD) containing genes are found in a relatively tight cluster on chromosome I, raising the possibility of a putative functional RD gene cluster in $D$. melanogaster.
\end{abstract}

Results: To investigate the possibility of such a gene cluster, orthologues of the Drosophila melanogaster RD genes were identified in several endopterygotan insects, two exopterygotan insects and two non-insect arthropods. In all insect species four RD genes were identified and orthology was assigned to the Drosophila sequences by phylogenetic analyses. Although four RD genes were found in the crustacean $D$. pulex, orthology could not be assigned to the insect sequences, indicating independent gene duplications from a single ancestor following the split of the hexapod lineage from the crustacean lineage.

In insects, two chromosomal arrangements of these genes was observed; the first a semi-dispersed cluster, such as in Drosophila, where lozenge is separated from the core cluster of three RD genes often by megabases of DNA. The second arrangement was a tight cluster of the four RD genes, such as in Apis mellifera.

This genomic organisation, particularly of the three core RD genes, raises the possibility of shared regulatory elements. In situ hybridisation of embryonic expression of the four RD genes in Drosophila melanogaster and the honeybee A. mellifera shows no evidence for either spatial or temporal co-linearity of expression during embryogenesis.

Conclusion: All fully sequenced insect genomes contain four RD genes and orthology can be assigned to these genes based on similarity to the $D$. melanogaster protein sequences. Examination of the genomic organisation of these genes provides evidence for a functional RD gene cluster. RD genes from non-insect arthropods are also clustered, however the lack of orthology between these and insect RD genes suggests this cluster is likely to have resulted from a duplication event independent from that which created the insect RD gene cluster. Analysis of embryonic RD gene expression in two endopterygotan insects, $A$. mellifera and $D$. melanogaster, did not show evidence for coordinated gene expression, therefore while the functional significance of this gene cluster remains unknown its maintenance during insect evolution implies some functional significance to the cluster. 


\section{Background}

Runt-domain (RD) containing proteins are transcriptional regulators that are able to activate or repress transcription dependent on the biological context of the cell [1] and are involved in cell fate specification and cell differentiation during development in metazoans [2]. The RD, a DNA binding domain, consists of a 128 amino acid motif that mediates DNA binding, heterodimerisation with a $\beta$-subunit (similar to the mammalian CBF $\beta$ proteins) [3] and also nucleotide binding [4] Outside of the $\mathrm{RD}$ itself, the only conserved motif in this protein family is a C-terminal pentapeptide (VWRPY, or I/LWRPF) which is thought to mediate the interaction with the transcriptional co-repressor Groucho [5].

The number of RD genes reported in metazoan genomes varies, and it is generally acknowledged that the RD genes have been independently duplicated in the chordate and insect lineages [6,7]. Studies of sea urchin (Strongylocentrotus purpuratus) runt and the mammalian runt orthologues Runx1, Runx2 and Runx3 have shown that RD proteins play an essential role in actively cycling cells and regulate cell proliferation prior to terminal differentiation [8]. All three RD proteins in humans are associated with disease, particularly cancer. Runx1, for example, is required for haematopoiesis and mutations in this gene cause leukaemia. Runx2 is required for bone development, and Runx3 for growth and differentiation of the gastric epithelium [8]. The Caenorhabditis elegans and S. purpuratus runt genes are also expressed in the gut $[9,10]$, implying that runx3 may be the ancestral runx gene in chordates [2], although this is not supported by molecular data [6].

The number of RD genes found in ecdysozoan genomes also varies, with a single RD gene in C. elegans, and three in the mosquito Anopheles gambiae [6]. In Drosophila, four $\mathrm{RD}$ genes are present in the genome: runt and lozenge, which have been very well characterised and two uncharacterised RD containing genes, CG42267 (previously CG15455) and CG34145 [6].

In Drosophila, runt is involved in many developmental processes, including embryonic segmentation $[11,12]$, sex determination $[13,14]$ and neurogenesis $[15,16]$. Runt is initially expressed in a broad domain in the centre of the syncitial blastoderm embryo regulated by bicoid $[17,18]$ and tailless [17], and is required for activation of sex-lethal, the major switch in Drosophila sex-determination [13]. Runt is a pair-rule gene but also has gap gene like properties [19]. During segmentation runt is expressed in the blastoderm with a dual segment periodicity, becoming expressed in seven stripes of cells. The expression of each of the seven stripes appears to be regulated independently by the gap genes, but possibly also by the maternal coordinate gene bicoid [17]. Other pair-rule genes, particularly hairy, act to maintain the runt expression pattern $[17,18]$. Later in the segmentation cascade runt is expressed with segmental periodicity, resulting in the formation of the secondary stripe pattern. This pattern is dependent on all of the pair-rule genes except sloppy-paired [17]. Following segmentation runt is expressed in a subset of cells in the developing nervous system and determines cell fate in specific neural lineages [16].

The other well-characterised Drosophila RD protein, lozenge, also has roles in several developmental processes. It has been shown to function in the development of antennae and tarsal claws, as well as haematopoiesis and female fertility [20]. Lozenge was first identified on the basis of its role in eye development and is critical for the differentiation of both photoreceptor neurons and non-neuronal cone cells $[21,22]$

Gene clusters, such as the Hox gene complex, are often ancient and are found in animals separated by hundreds of millions of years of evolution. While gene clustering as a phenomenon appears more common in genomes of higher complexity, for instance in mammalian as compared to insect genomes, very ancient clusters such as those involving the wnt family of genes [23] and the Fox gene transcription factors [24] have been reported across evolution. In the well-studied cases of the Hox, Wnt and Fox clusters, genes within the cluster show coordinated expression during embryogenesis, implying the genes may be regulated by shared cis-regulatory elements, which invoke stabilising selection to maintain the gene cluster. For the majority of gene clusters, however, it is not clear whether the genomic organisation is maintained as a result of functional constraints, or whether it is merely by chance that no chromosomal rearrangements disrupting the complex have become fixed.

It has been previously noted that of the Drosophila RD genes, runt, CG34145 and CG42267 are closely linked on chromosome 1 [6], and are likely to have evolved as a result of tandem gene duplication. The close linkage of these genes may be due to the recent evolution of multiple RD genes in Drosophila or alternatively may indicate the presence of functional constraints that retain these genes in close proximity. It is possible to distinguish between these possibilities by comparison of the genomic organisation amongst species that are sufficiently diverged to have had numerous chromosomal rearrangements become fixed, resulting in a shuffling of both the order and spacing of genes.

A comprehensive survey of runt domain containing genes in a wide range of arthropods is now possible due to the availability of genome sequence for several key species. Here we identify the RD genes in the genomes of several 
endopterygotan insects, including the twelve Drosophila species [25], Apis mellifera (the honeybee) [26], Nasonia vitripennis (the jewel wasp) [27], Tribolium castaneum (the red flour beetle) [28]Bombyx mori (the silkmoth) [29] and Aedes aegypti (the yellow fever mosquito) [30]. The genomes of two fully sequenced exopterygotan insects, Acyrthosiphon pisum (the pea aphid) [31] and the Pediculus humanus (the human body louse) [32] were also included in this analysis. In addition two non-insect arthropod genome sequences are now available: the crustacean Daphnia pulex [33] and the chelicerate Ixodes scapularis [34]. We examine the phylogenetic relationships and genomic organisations of the RD genes in these organisms, and present evidence for a putative conserved runt complex in insects. To determine if the close genomic proximity of these RD genes in insects relates to functional conservation of regulatory elements, the embryonic expression profiles of the four RD genes were examined in $D$. melanogaster and the hymenopteran insect A. mellifera.

\section{Results \\ Identification of a runt gene complex in Drosophila species}

Three of the four RD genes in D. melanogaster are clustered within $163 \mathrm{~kb}$ on chromosome 1 and are likely to have arisen by tandem gene duplication [6]. There is very tight linkage between CG42267 and CG34145, but there are three annotated functionally unrelated genes that lie between CG34145 and runt. Lozenge is separated from the other three genes by a distance of over $11 \mathrm{Mb}$.

To determine whether the genomic organisation of this 'runt complex' is the result of functional constraint or has been maintained purely by chance, we utilised the recent release of the twelve Drosophila species genome sequences [25]. These species are approximately 40 - 60 million years diverged [35], and extensive gene shuffling within the chromosome arms has occurred even between moderately diverged genomes such as D. melanogaster and $D$. yakuba $[25,36]$.

Four RD genes were identified in each of these Drosophila genomes; in some cases the gene prediction was incomplete and complete predictions were obtained using Augustus [37]. In all cases the presence of both the RD and C-terminal VWRPY motif was confirmed. Identity was then assigned to these orthologues based on homology to the D. melanogaster protein sequences, as inferred by Bayesian phylogeny (Fig. 1) [see Additional file 1 for multiple sequence alignment].

The orthologues of CG34145, CG42267 and Runt are all found tightly linked (within $140-225 \mathrm{~kb}$ ) in all 12 Drosophila species. In contrast, the orthologue of lozenge is usually present on the same chromosome but not closely linked to the other three RD genes. In species where scaffolds have been assigned to chromosome groups, this complex falls on the X chromosome (chromosome 1 in $D$. melanogaster).

The number and identity of intervening genes is not well conserved (Fig. 2), although Cyp6v1 is always found between CG34145 and runt. Hydra and CG1835 are found between these two genes in the melanogaster sub-group, with the exception of $D$. sechellia where both genes are localised to an orphan scaffold (scaffold 600) which is likely to be an assembly error. CG1835 is also found in a conserved position in D. willistoni. There is also an unannotated gene likely to fall within this region in D. melanogaster that shows homology to D. simulans GD17499, this gene is found in all members of the melanogaster group but is unannotated in most (Fig. 2). It is possible that this sequence does not produce an mRNA transcript, although there is EST evidence in D. melanogaster to suggest that it does (BK003230). Despite the variation in number of intervening genes, the approximate spacing between the three genes is well conserved; with $\sim 100 \mathrm{~kb}$ between runt and CG34145 (in all species except D. sechellia, where the distance is $20 \mathrm{~kb}$ ) and $\sim 168 \mathrm{~kb}$ between CG23415 and CG42267. The orientation of transcription is also conserved; orthologues of CG42267 and runt are transcribed from the sense strand, while CG34145 is transcribed from the anti-sense strand. The transcriptional orientation of lozenge is not well conserved, but it is transcribed from the sense strand in the majority of species, except D. erecta and D. virilis, possibly resulting from independent genome inversion events in these species.

Together, the phylogenetic and genomic organisation supports the duplication of the RD genes prior to the radiation of the Drosophila lineage, and the resultant cluster of $\mathrm{RD}$ genes shows remarkable stability since the divergence of Drosophila species 60 million years ago [35].

\section{Identification of a runt gene complex in other insect species}

A survey of other fully sequenced insect genomes [26$28,31,32,38]$, shows that aspects of this runt gene complex are conserved, not only in the endopterygotan insects A. mellifera, A. aegypti, B. mori and T. castaneum, but also in the exopterygotan insects $P$. humanus (a Phthiapteran) and A. pisum (a Hemipteran). In all of these insects four RD genes were identified, and phylogenetic analyses (Fig. 3 ) were used to assign orthology to these genes. Where RD genes were incompletely or incorrectly annotated, particularly in the genomes of T. castaneum, A. aegypti, A. pisum and $B$. mori, annotation was performed manually with the assistance of Augustus [37] and GenomeScan [39], in all cases the presence of both the RD and C-terminal VWRPY motif was confirmed (the C-terminal pentapeptide in $A$. 


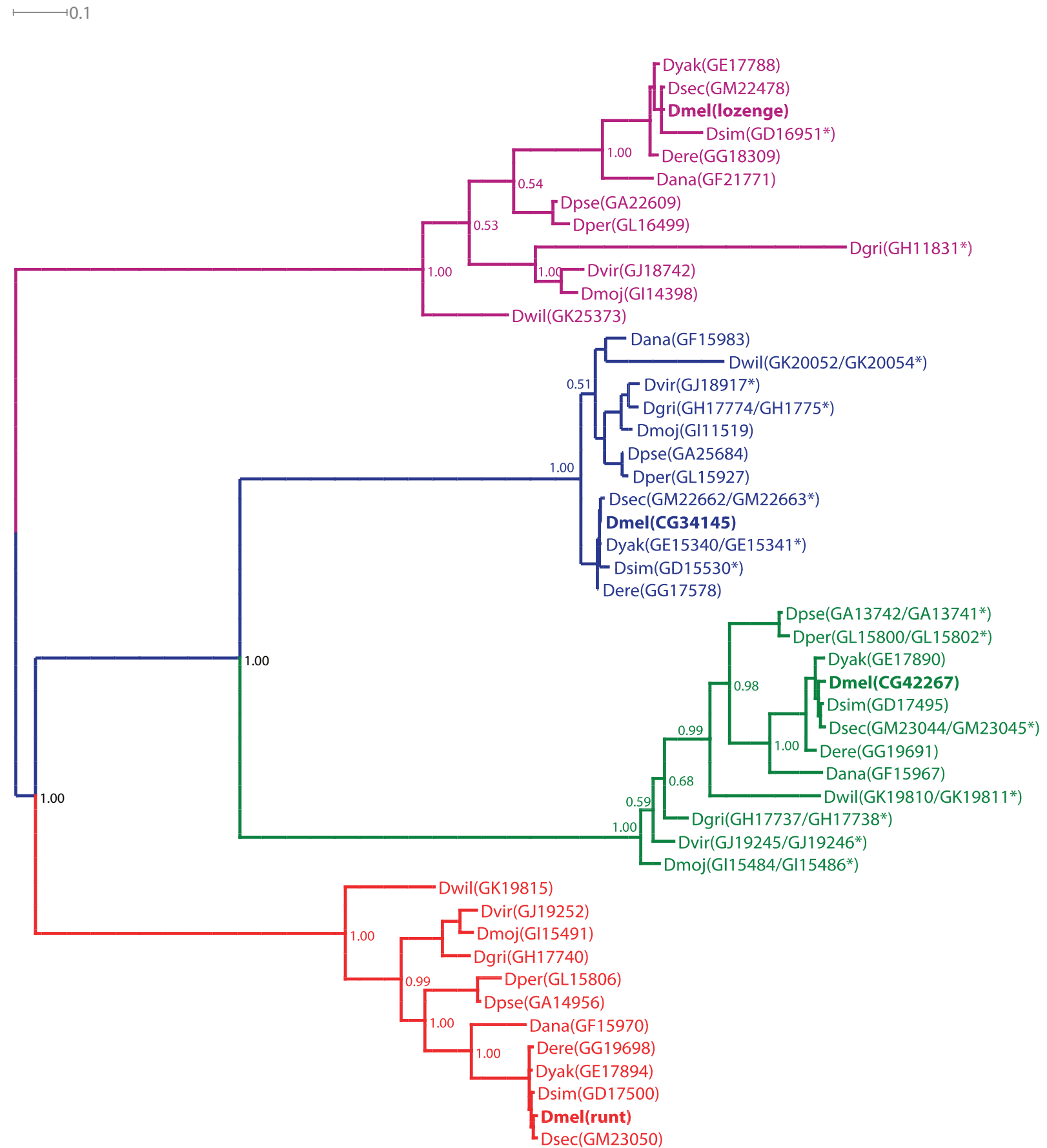

Figure I

Phylogeny and genomic organisation of Drosophila runt domain protein sequences.

An unrooted Bayesian phylogeny of Drosophila full-length RD protein sequences. Phylogeny was constructed using MrBayes [67] under the Jones amino acid substitution model. Posterior probabilities are shown on internal branches. The RD proteins are subdivided into the four established orthologue groups. Species names are abbreviated as follows: Dmel $=D$. melanogaster, Dsim $=D$. simulans, Dsec $=D$. sechellia, Dyak $=D$. yakuba, Dere $=D$. erecta, Dana $=D$. ananassae, Dpse $=D$. pseudoobscura, Der $=D$. persimilis, Dwil $=D$. wilistoni, Dmoj $=D$. mojavensis, Dvir $=D$. virilis, Dgri $=D$. grimshawi and species names are followed by the FlyBase accession numbers. Asterisks indicate protein sequences that have been manually annotated. Orthologue groups are colour coded, the red group shows homology to the Drosophila runt protein, the purple to the Drosophila lozenge protein, the green to the Drosophila CG42267 protein and the blue to the Drosophila CG34I45 protein. 


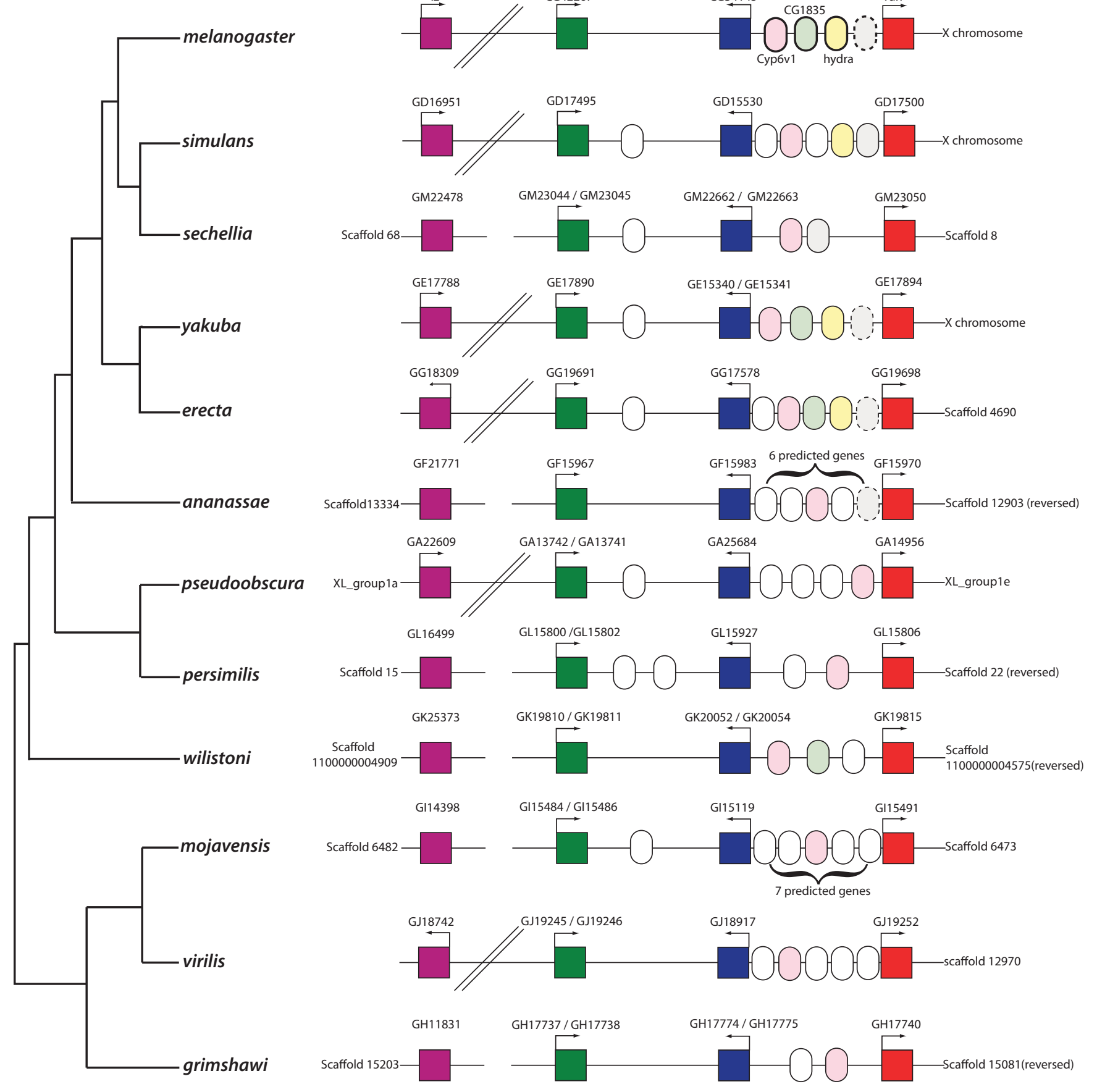

Figure 2

Genomic organisation of the Drosophila RD gene clusters. Orthologous genes are colour coded consistent with the phylogeny in Fig. I. In D. melanogaster Cyp6v I, CG I 835 and hydra are annotated to fall between CG34 I 45 and runt, Drosophila orthologs of these genes are coloured pale pink, pale green and pale yellow, respectively. A fourth gene, which is unannotated in $D$. melanogaster (as denoted by the dashed lines) is shown in light grey. 
pisum XP001950158 is modified to IWRPF, and followed by an additional 5 amino acids prior to the stop codon). Proteins that were manually annotated in this way are indicated with an asterisk on the phylogeny presented in Fig. 3 [see Additional file 2 for multiple sequence alignment and phylogeny for endopterygotan insects, and Additional file 3 for multiple sequence alignment for both endopterygotan and exopterygotan insects].

It has been generally asserted that the RD gene family has derived from serial duplication of a single ancestral gene [6]. The structure of the insect RD gene phylogeny presented in Fig. 3, indicates that runt is ancestral to the other three RD proteins, and has been duplicated twice; one copy became the runt gene, while the second was further duplicated to evolve the CG34145 and CG42267 genes.

Comparison of the genomic organisation of these RD genes (Fig. 4) confirms a tight cluster of all four RD genes in $B$. mori and the most basally branching endopterygotan insect, A. mellifera [40]. In A. mellifera there are no inter- vening genes and the total cluster spans only $158 \mathrm{~kb}$, however the $B$. mori cluster spans $330 \mathrm{~kb}$ and there are 5 annotated genes falling between BmCG42267 (BGIBMGA008905) and BmCG34145 (BGIBMGA008970/ 008971). Surprisingly, a very tight cluster of all four RD genes can also be found in the exopterygotan insect $P$. humanus, where all four genes are found within $70 \mathrm{~kb}$, with no intervening genes.

Data for A. aegypti and A. pisum were inconclusive due to gaps in the genome assemblies, but the orthologues of both runt and CG34145 were found on the same contig in close proximity, consistent with that seen in the other insects. In all cases the transcriptional orientation of these three core RD genes is completely conserved.

Interestingly, in the species where the four $\mathrm{RD}$ genes are tightly linked (A. mellifera, B. mori and P. humanus), the orthologue of lozenge lies proximal to the orthologue of runt. This contrasts to the remainder of species where loz-

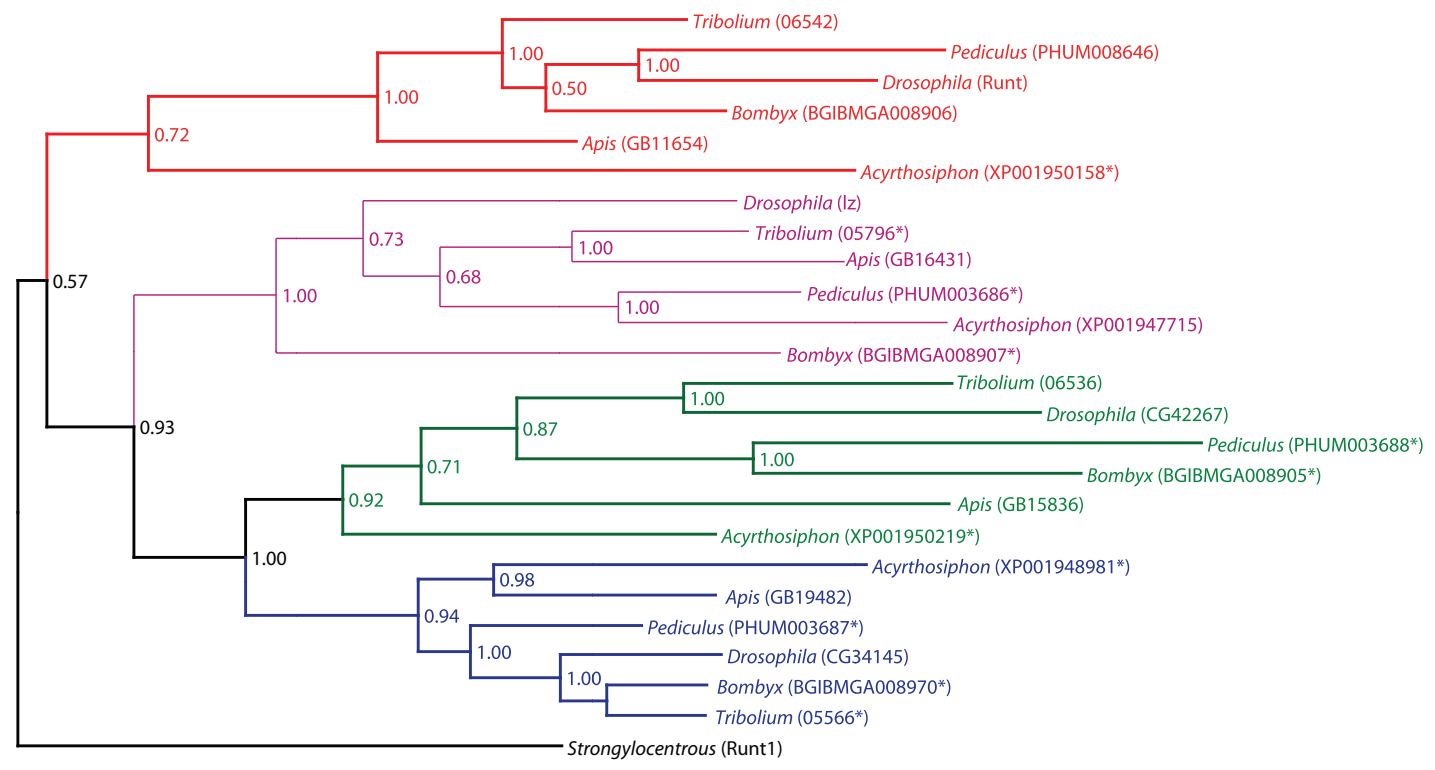

Figure 3

Phylogeny of the runt domain protein sequences in endopterygotan and exopterygotan insects. Rooted Bayesian phylogeny of full-length runt protein sequences. Phylogeny was constructed in MrBayes; posterior probabilities are shown on internal branches; the tree was rooted with runt from the sea urchin S. purpuratus. Orthologue groups are colour coded, the red group shows homology to the Drosophila runt protein, the purple to the Drosophila lozenge protein, the green to the Drosophila CG42267 protein and the blue to the Drosophila CG34I45 protein. Asterisks indicate protein sequences that have been manually annotated. 


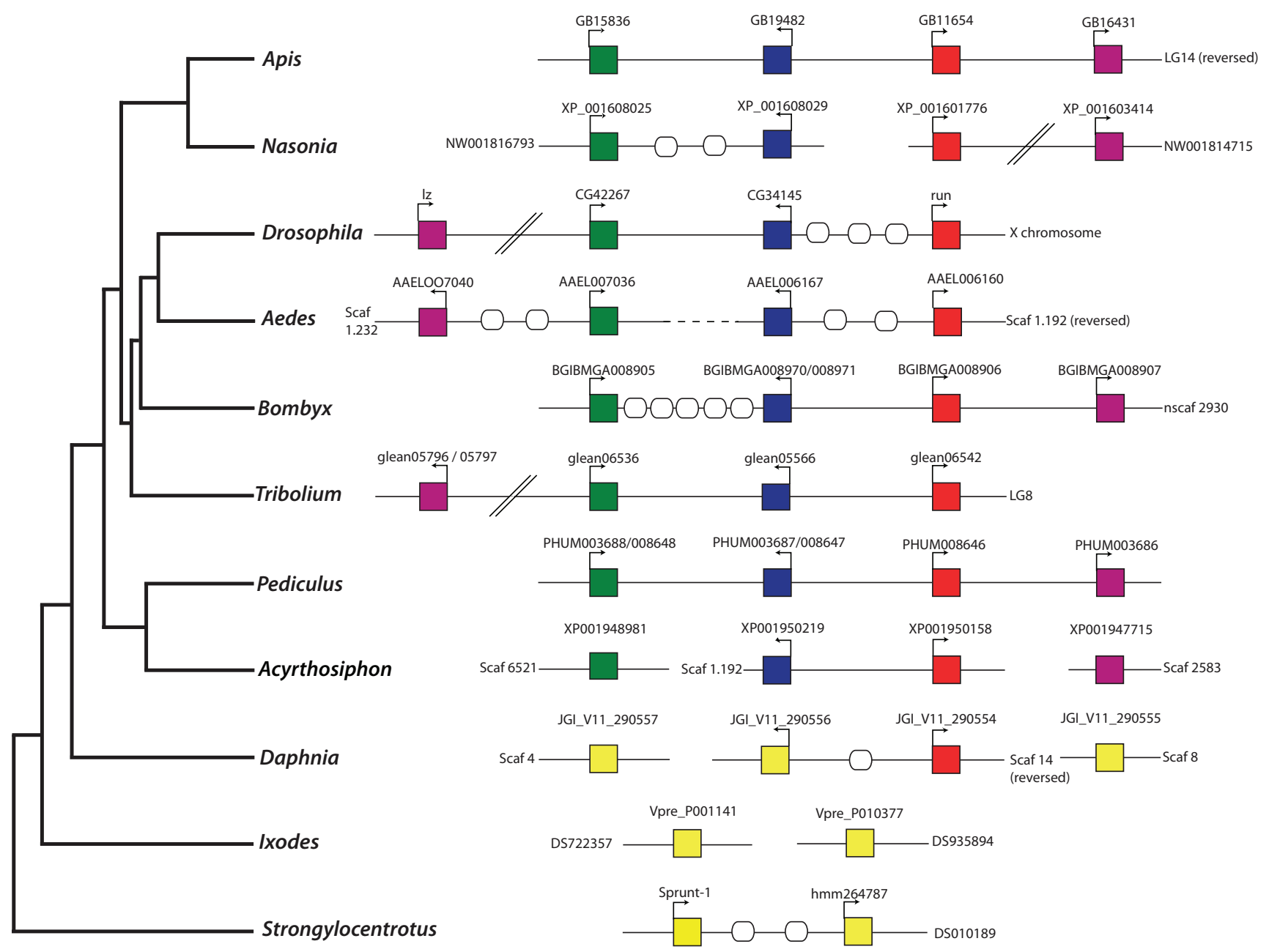

Figure 4

Genomic organisation of the RD genes in insect and non-insect arthropod species. Genomic organisation of the RD genes in insect and non-insect arthropod species. Orthologous genes are colour coded, the red group shows homology to the Drosophila runt protein, the purple to the Drosophila lozenge protein, the green to the Drosophila CG42267 protein and the blue to the Drosophila CG34I45 protein.

enge often lies distal to the RD cluster, separated by a megabase or more.

\section{Expression of the RD genes: comparison of Drosophila and $A$. mellifera}

The persistence of an intact RD gene cluster in all insects examined, despite more than 400 million years of evolution, and significant changes in life history and developmental modes, implies some functional relevance to the organisation of these genes. Analysis of gene expression patterns has previously provided important insight into the nature of selective forces acting on gene clusters [41]. The expression patterns of runt and lozenge are well-understood in D. melanogaster, but the expression patterns of the other two RD genes remains uncharacterised. As the Drosophila RD complex shows evidence of dispersal, of lozenge in particular, we also chose to examine the expression patterns of all four RD genes in the basally branching endopterygotan insect, A. mellifera, which possesses a tight cluster of all four RD genes (Fig. 4). 
In situ hybridisation of Drosophila and A. mellifera embryos was used to compare expression domains of the four RD mRNAs. Drosophila runt (Fig. 5a) is expressed early in development in a broad domain, then in a dualsegment periodicity in embryonic stages $4-6$, and later in a segmental periodicity following gastrulation, as previously reported [42]. A. mellifera runt is expressed in a similar pattern (Fig. 5b), in stripes across the embryo that form in an anterior to posterior succession in a pattern that is typical of A. mellifera pair-rule genes [43].

The expression of Drosophila lozenge (Fig. 5g) is not consistent with that previously reported; a previous study demonstrated that by embryonic stage 7 lozenge expression is restricted to the anterior of the embryo in a subpopulation of prohaemocytes, which terminally differen-

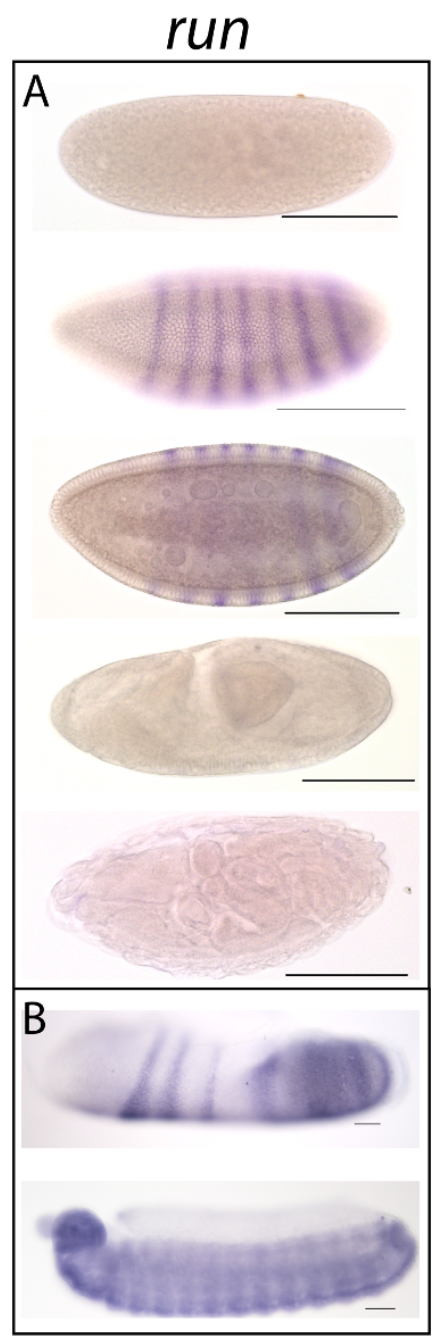

GB11654
CG34145

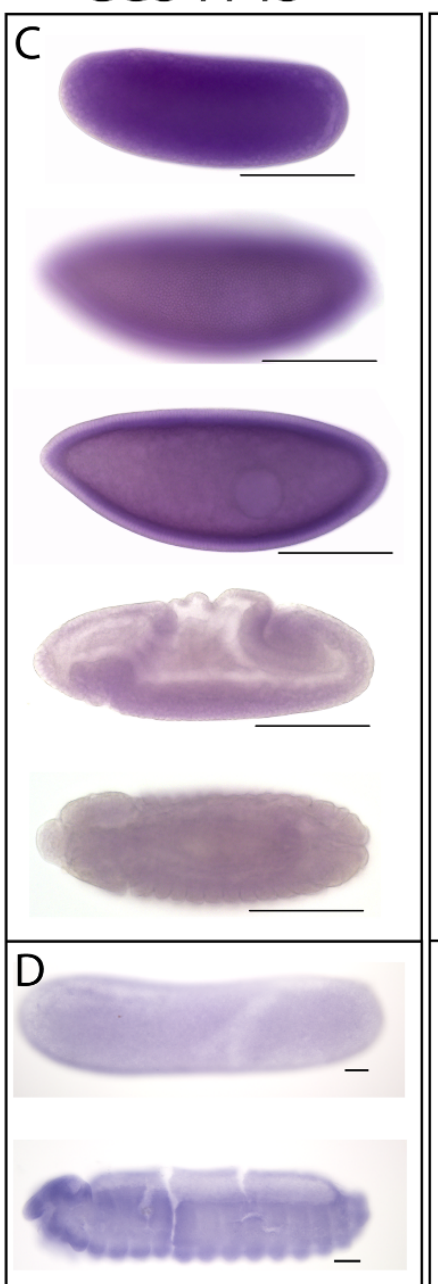

GB19482
CG42267

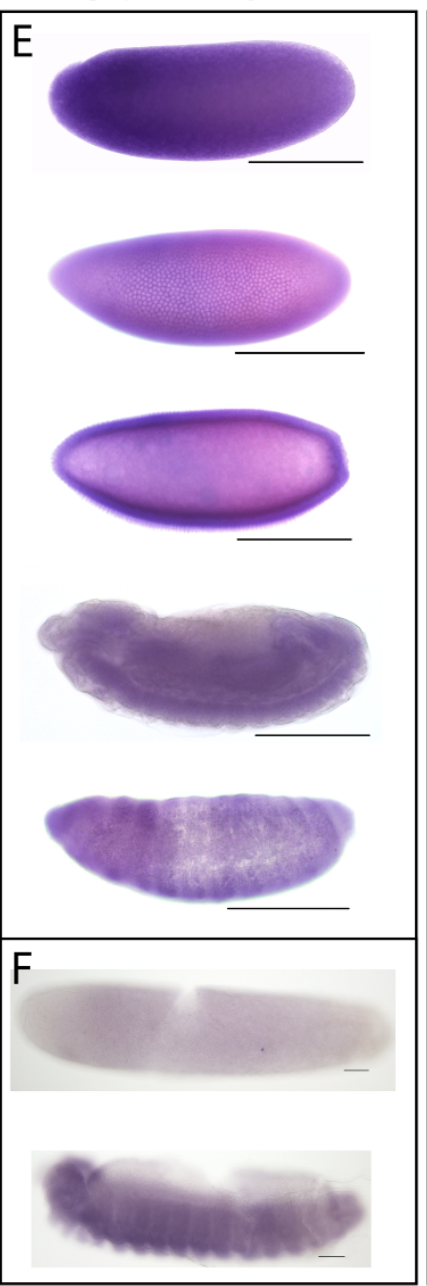

GB15836

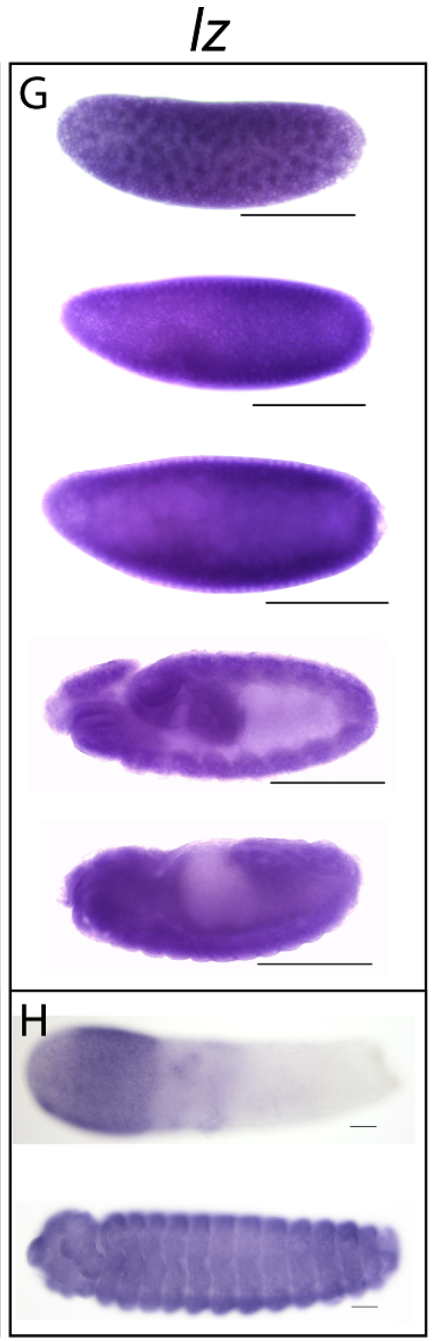

GB16431

\section{Figure 5}

Expression of the RD mRNAs in Drosophila and A. mellifera embryos. Expression of RD gene RNA in Drosophila and Honeybee embryos using in-situ hybridisation. Genes arranged by orthology based on Fig. 3. A, C,E, and G Drosophila, scale bars $=100 \mu \mathrm{m} \mathrm{B}, \mathrm{D}, \mathrm{F}$ and $\mathrm{H}$ honeybee, scale bars $=50 \mu \mathrm{m} \mathrm{A}$ ) run (stage 2, 5 (two focal planes), 9 and I6) is in seven stripes in blastoderm embryos, no maternal expression is seen and expression is low in later development. B) GB I l654 (stages 5 and 9) is expressed in stripes in the blastoderm, and is expressed widely in later embryos. C) CG34I 45 (stages 2, 5 (two focal planes), 7 and I5) is expressed maternally and then ubiquitously until stage I4. D) GB/9482 (stage 5 and 9) is expressed weakly in stage 5 embryos but ubiquitous later. E) CG42267 (stage 2, 5 (two focal planes), I 3 and 16) is expressed maternally and then ubiquitously. F) GB I5836 (stages 5 and 9) is expressed ubiquitously after blastoderm stages. G) Iz (stages 3, 5 (two focal planes), I I and I5) is maternally provided localised to nuclei and ubiquitous by stage 5. H) GBI643/ (stages 5 and 9) is expressed in the anterior of blastoderm embryos and ubiquitously by stage 9 . 
tiate to form crystal cells (which are involved in innate immunity and wound healing) by embryonic stage 10 [44]. In this study lozenge mRNA is initially maternally provided and is localised to nuclei at early stages, expression later in development is ubiquitous. This difference in expression patterns is probably because the previous study used an enhancer fragment linked to a reporter gene to determine the expression pattern. A. mellifera lozenge (Fig. 5h) expression is detected very early (stage 1 ) in the anterior region of the embryo and is then detected in a distinct cell type, that develop from head mesoderm (Fig. $5 \mathrm{~h}$ ). These may be differentiating crystal cells, which develop from the head mesoderm in Drosophila [45]. By stage 9 (just prior to hatching), Amlz is expression is detected throughout the embryo (data not shown).

The embryonic expression of the other two RD genes in Drosophila, CG34145 (Fig. 5c) and CG42267 (Fig. 5e), have not been previously described. At all embryonic stages expression of both of these genes is ubiquitous, with in situ hybridisation of imaginal discs also showing no evidence of localised expression (data not shown). Both AmGB11654 and AmGB19482, the A. mellifera orthologues of these genes, are also expressed ubiquitously throughout late stages of embryonic development (Fig. 5d and 5f).

While clustered genes often show coordinated expression, neither the Drosophila nor A. mellifera RD genes (Fig. 5) demonstrate strong coordination of either temporal or spatial expression during embryonic development.

\section{The evolution of RD proteins in metazoa and arthropods}

The presence of a runt domain cluster (Fig. 4) in all insect species examined, together with the phylogenetic data presented in Fig. 3, suggests that the duplication of the RD genes occurred prior to the divergence of the insect lineage into these two branches. In order to examine the evolution of these RD genes, and this cluster in a wider context, the genome sequence of two non-insect arthropods were utilised. Examination of the genome sequences of the crustacean D. pulex and the chelicerate I. scapularis clearly demonstrates multiple RD genes in both of these species, contradicting previous assertions that non-insect arthropods have only a single RD gene [7]. The water flea $D$. pulex has four RD genes, and two of these fall on the same scaffold (Fig. 4). In contrast I. scapularis only has two RD genes, and at present these fall on two independent scaffolds. Extensive searches of the I. scapularis genome have been unable to identify any further orthologues, however this could be due to the preliminary nature of the genome assembly. Two RD genes have also been identified in the spider Cupiennius salei [46], and one in the two-spotted spider mite, Tetranychus urticae [47].
This presence of multiple $\mathrm{RD}$ genes in the genomes of non-insect arthropods raises the possibility that the duplication of the RD genes occurred prior to the radiation of arthropod species and that multiple RD genes and their genomic organisation were present in the last common ancestor of arthropods. To examine this possibility phylogenetic analyses were carried out using the $\mathrm{RD}$ domain sequence from the proteins of known RD genes. Representatives from several non-arthropod metazoan clades were selected, including the basally branching non-bilaterian Trichoplax adhaerens [48], the cnidarian Nematostella [49], and the RD genes from two sequenced lophotrochozoan species, the gastropod snail Lottia gigantea, and the polychaete Capitella (Fig. 6) [see Additional file 4 for multiple sequence alignment].

Phylogenetic analysis indicates that the duplication of the RD genes has occurred independently in the deuterostome and arthropod lineages, and that the duplication events that gave rise to the multiple vertebrate runx genes, have occurred independently of the duplications giving rise to the multiple RD genes in the sea urchin, S. purpuratus. Somewhat surprisingly however, the phylogeny also indicates independent duplication events have given rise to the chelicerate RD genes and the crustacean/insect RD genes. The phylogeny indicates a single origin for chelicerate RD genes independent of the duplications that gave rise to the RD genes in Daphnia and in insects. Supporting this is the expression pattern of the RD gene cloned from Tetranychus urticae (the two-spotted spider mite), which clearly suggests a role for Tu-run in segmentation, but also in limb specification [47], a role not seen for either the A. mellifera or Drosophila orthologues of runt, but consistent with expression of Cs-runt in C. salei.

Interestingly, a single D. pulex gene (Dp290554) groups with the clade of insect RD proteins, while the other three $D$. pulex genes form an independent clade. This seems to indicate that runt is the ancestral protein in crustaceans and chelicerates. However, the crustacean/insect clade on the whole is not well resolved, as by necessity, this phylogeny only included 125 amino acids of the RD as two of the $D$. pulex sequences are incorrectly annotated and are missing 10 amino acids of the $\mathrm{RD}$, thus reducing the informational content of the alignment.

Bayesian phylogeny of the full RD from arthropod species, minus the truncated D. pulex sequences (Fig. 7) [see Additional file 5 for multiple sequence alignment], supports the independent duplication of $\mathrm{RD}$ genes in chelicerates and in crustaceans and insects. As before Dp290554 groups reliably with the insect runt $\mathrm{RD}$ sequence, providing evidence for runt being the ancestral $\mathrm{RD}$ protein in these species, which was suggested (albeit 


\section{INSECTS/CRUSTACEANS}

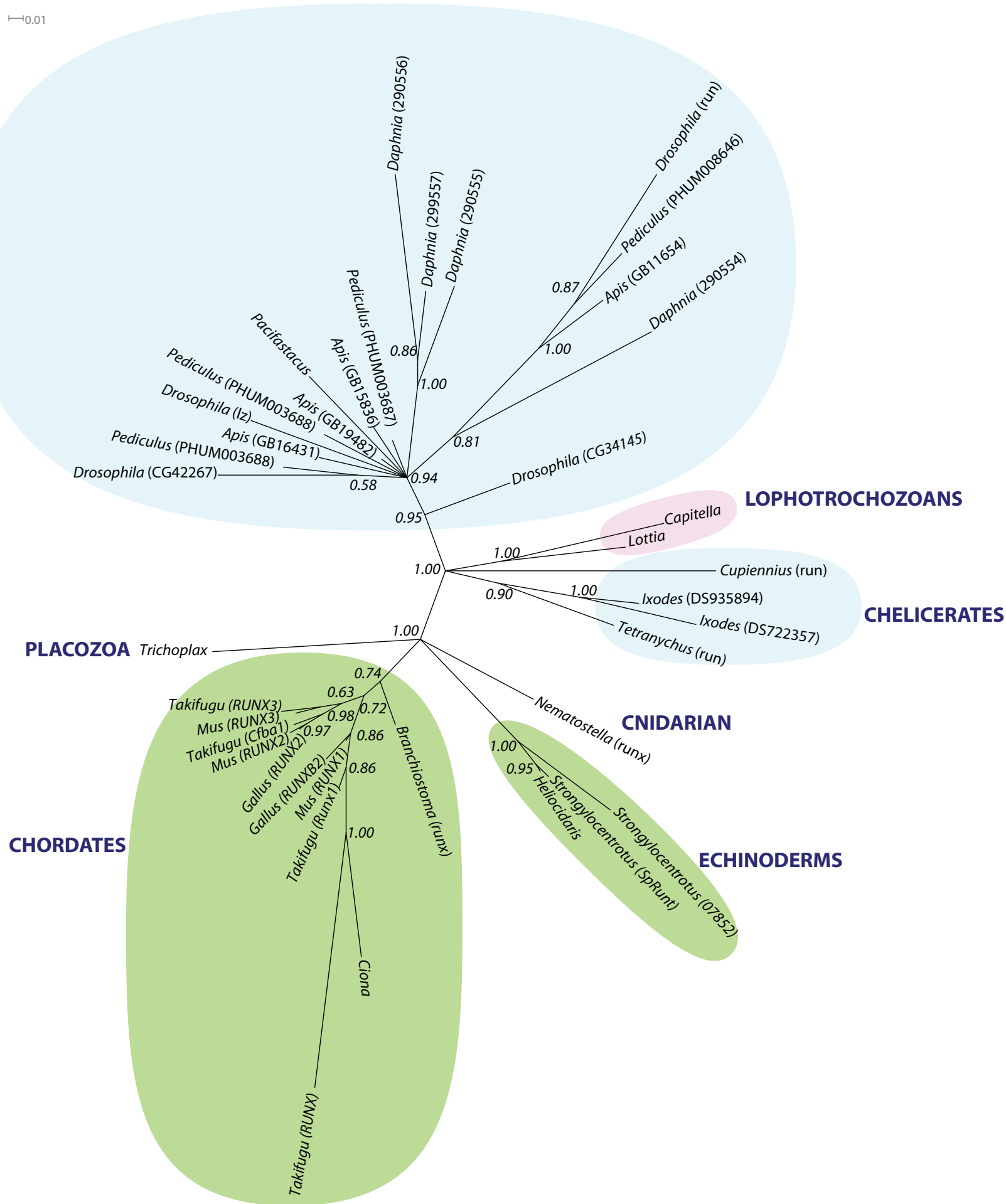

\section{Figure 6}

Phylogenetics of the runt-domain protein families in Metazoan species. Phylogeny of metazoan Runt/runx proteins based on alignment of I 25 amino acids of their RD sequences. Unrooted phylogram constructed using MrBayes [67] under the Jones amino acid substitution model. Deuterostome species are highlighted in green, arthropod species in blue and lophotrochozoans in pink. Posterior probabilities are shown on internal branches. 


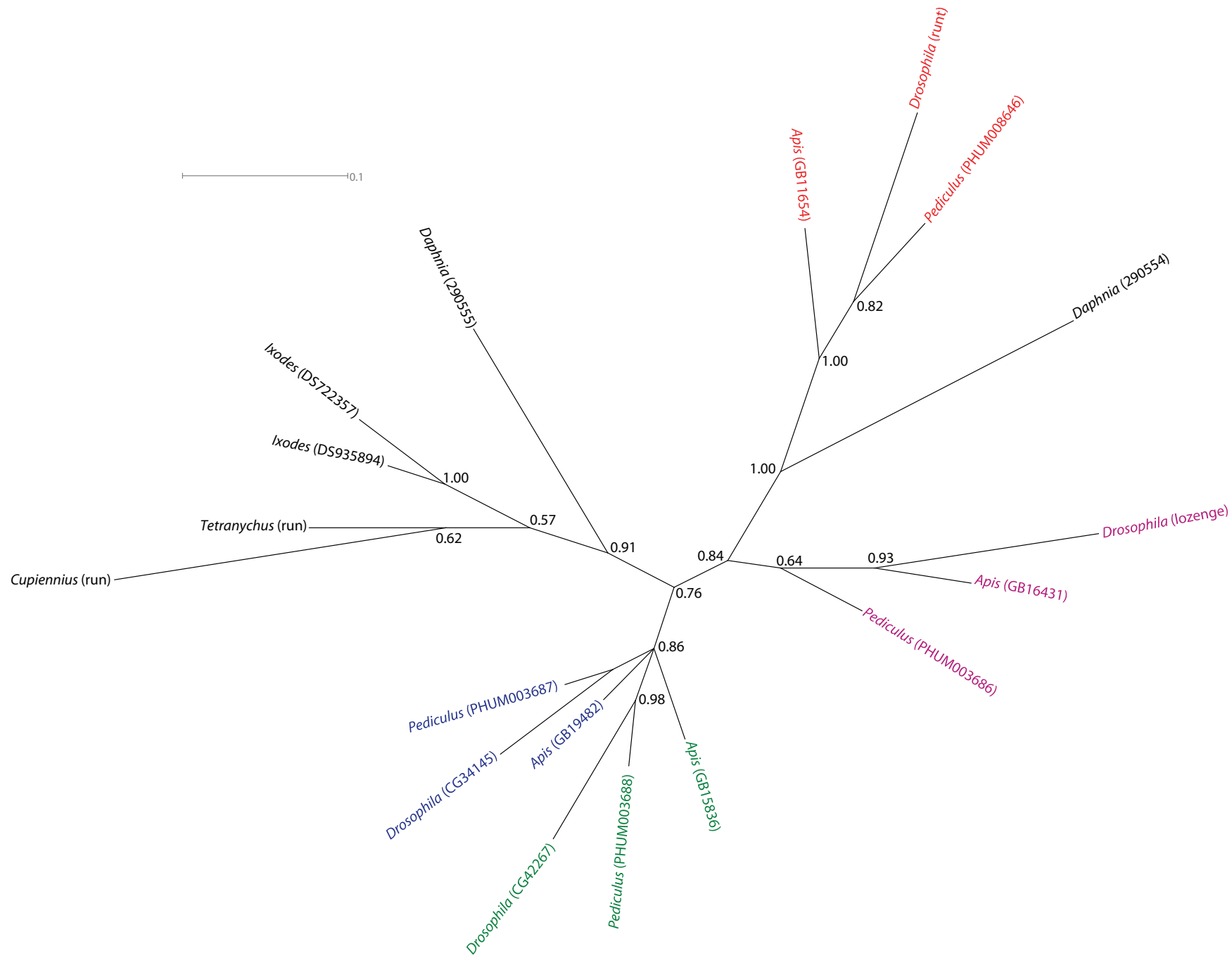

Figure 7

Phylogenetics of the runt-domain protein families in Arthropods. Phylogeny of arthropod RD proteins based on alignment of the full-length RD sequences (I 33 amino acids). Unrooted phylogram drawn from Bayesian phylogenetic analysis of RD domain sequences under the Jones amino acid substitution model. The insect proteins are colour coded based on homology to the Drosophila sequences as in Figs. I and 3. Posterior probabilities are shown on internal branches.

weakly) by the phylogeny of endopterygotan and exopterygotan full-length protein sequences in Fig. 3.

\section{Discussion}

In this study we identified four RD genes in the twelve sequenced Drosophila genomes [25] and in several nondipteran endopterygotan insects including A. mellifera, A. aegypti, B. mori, and T. castaneum. Four RD genes were also identified in the exopterygotan insects, $P$. humanus and $A$. pisum, and the crustacean D. pulex. Two different arrangements of RD genes were observed in these species: a semidispersed cluster was seen in Drosophila species and in $T$. castaneum; and an intact, tight cluster with few or no intervening genes was seen in A. mellifera, B. mori and $P$. humanus. In the other species examined, the genome assembly was fragmented such that one or more RD genes was localised to a different contig and thus no assertions about cluster organisation could be made. In species with an intact RD gene cluster, lozenge was found to be located proximal to runt, while in the species with a semi-dispersed cluster lozenge was often localised to the same chromosome, but separated from the core cluster by megabases of DNA. The genomic organisation of the remaining three $\mathrm{RD}$ genes, including gene spacing and transcriptional orientation, was remarkably conserved.

The number and identity of intervening genes in this cluster varies; between two and seven genes lie between the 
CG34145 and runt orthologues in Drosophila species, including Cyp6v1. Cyp6v1 is a P450 enzyme, and is part of a large gene family that is prone to duplication [50]. Hydra and CG1835 are localised between CG34145 and runt in D. melanogaster, D simulans, D. yakuba and D. erecta, these two genes are localised to an orphan scaffold in $D$. sechellia (scaffold 600), but this is likely a problem with the genome assembly. Hydra is found exclusively in the melanogaster sub-group, with no orthologues found in any other dipterans or insects (data not shown), suggesting recent evolution of this gene. Conversely CG1835 is also found in D. willistoni suggesting at least two independent losses of this gene (in the obscura group and D. ananassae) A fourth gene is found in this region in the melanogaster species group, however this gene is unannotated in $D$. melanogaster and therefore may represent a conserved regulatory sequence rather than a protein coding gene. CG42267 and CG34145 are in much tighter linkage, and the majority of Drosophila species have either one or no intervening genes in this region, other than $D$. persimilis which has three. Based on established taxonomic relationships between Drosophila species it is likely that there has been multiple gains or losses of genes in these genomic regions, although this effect may be exaggerated due to inaccuracies in the genome assemblies or gene prediction models. In all but one non-dipteran insect species, no intervening genes were identified in the RD gene cluster, the only exception being in $B$. mori where five annotated genes fall between BmCG42267 and BmCG34145. The presence of intervening genes in the Dipteran RD cluster, seemingly unrelated in either expression or function, is consistent with the RD complex being considered a genomic regulatory block [51] and is reminiscent of the Hox complex in Drosophila, where invasion of cuticle genes into the ANT-C complex between labial and proboscipedia has been attributed to the accelerated rate of evolution in this lineage, and may have contributed to splitting of the Hox complex in Drosophila [52].

The presence of an intact cluster in the endopterygotan insects A. mellifera and B. mori, and the exopterygotan insect $P$. humanus, strongly supports the phylogenetic data that duplication of the RD genes occurred prior to the radiation of insect species, and implies that the ancestral insect genome had a very tight cluster of four RD genes. The semi-dispersed gene cluster seen in Drosophila and T. castaneum may indicate that the functional constraint holding lozenge in the gene cluster has been lost or modified in the Coleopteran and Dipteran lineages. The pattern of RD gene dispersal in these species is similar to that seen for the Fox family of transcription factors, where relaxation of selection on the cluster has resulted in cluster fragmentation [24]. The dissolution of such clusters also may be the result of accelerated genome evolution associated with Drosophila lineages [25]. However, the T. castaneum genome is not noted to have undergone particularly rapid evolution [53] and consistent with this, the T. castaneum genome has just a single Hox complex, with no evidence of dispersal [53]. A recent study has shown that while up to $91 \%$ of orthologues remain in synteny between $D$. melanogaster and the most diverged Drosophila species, D. virilis synteny is only $3 \%$ between $D$. melanogaster and $B$. mori, and $10 \%$ between D. melanogaster and A. mellifera [54]. This, along with the high observed rate of turnover of intervening genes within the RD cluster, would indicate that the conservation of genomic organisation seen for RD genes across insect genomes would be very unlikely to have occurred simply by chance, and is likely to have been maintained by selection, presumably in favour of retaining function.

Functionally related genes are known to have, in some cases, conserved genomic organisation [55]. In general, genes that have evolved as a result of tandem gene duplications tend to maintain a similar function to their parental copy due to sharing of the same regulatory elements [56], and this sharing of regulatory elements can facilitate the evolution of co-regulation [41]. To examine the possibility of co-regulation acting as a selective force to maintain the RD gene cluster, the expression of the four RD genes was examined in D. melanogaster (which houses a semi-dispersed RD cluster) and in A. mellifera (which houses an intact 'ancestral' RD cluster) as a means of investigating functional conservation of RD gene orthologues between these species. In situ hybridisation did not reveal any strong evidence for spatial or temporal co-regulation of RD gene orthologues during Drosophila or $A$. mellifera embryogenesis. Although it is possible that localization or expression of the protein (which would not be detected by in situ hybridisation) is altered independently of that of its mRNA. Three of the four RD genes are expressed ubiquitously in the blastoderm stage of embryogenesis in Drosophila, while the fourth, runt is expressed in a pair-rule pattern in seven stripes. Therefore the cluster of RD genes in insects could be driving co-regulation of expression at the blastoderm stage, while runt expression is negatively regulated by fushi tarazu [18], however this is not the case for A. mellifera. It is possible that overlapping rather than shared regulatory elements are responsible for the retention of the gene cluster. However, this scenario would also appear unlikely; while the runt enhancer in Drosophila covers a distance of approximately $14 \mathrm{~kb}$ [57], the intergenic distance between CG34145 and runt averages around $100 \mathrm{~kb}$ across Drosophila species, and the number of intervening genes in this region varies markedly, highlighting that this genomic region is free to evolve.

There has been much speculation about the origin of the $\mathrm{RD}$ proteins, and it is largely accepted that the RD genes 
have been independently duplicated in the deuterostome and protostome lineages $[6,7]$. However, the question of RD gene evolution in arthropods has not been addressed specifically. Phylogenetic analyses presented here support the notion of gene duplication prior to the radiation of insect species, as all insect RD proteins could be placed into four orthologue groups. However, two non-insect arthropods whose genomes have been sequenced completely, the chelicerate I. scapularis and the crustacean $D$. pulex, also have multiple $\mathrm{RD}$ genes, and phylogenetic analyses support the notion that these genes have been independently duplicated in insect and non-insect arthropod lineages. This phylogeny implies that the ancestor of arthropods had a single RD gene. However, gene conversion events are known to result in phylogenetic analyses that overestimate the incidence of gene duplication. This is an issue for linked genes, such as those generated by tandem gene duplication [58], and has resulted in spurious phylogenies for insect gene families [59].

The phylogeny presented here indicates that there have been multiple duplications of RD genes across bilaterian evolution, and as there is no evidence for the duplicated copies decaying into pseudogenes, these duplicated copies are likely to have undergone rapid divergence resulting in subfunctionalisation or neofunctionalisation of the protein functions. This kind of rapid divergence could be due to the chromosomal location of the ancestral gene, as it is known that areas of the genome are more prone to this phenomenon, such as the $\mathrm{X}$ chromosome in Drosophila, where the RD gene complex is localised [60]. Consistent with the hypothesis of rapid divergence following duplication, there is clear evidence for neofunctionalisation of the duplicated genes, in both ecdysozoan and deuterostome species. For instance, the $C$. elegans RD gene and one of the sea urchin $\mathrm{RD}$ genes, which are most closely related to mammalian runx3, are both expressed in the developing gut, a role not seen in the cnidarian Nematostella. This has led to the assertion that runx 3 may be the ancestral RD gene in chordates [2], although this hypothesis is yet to be supported by molecular data [6].

To date there has been no discussion about the evolution of RD genes in arthropods, and the phylogenetic analyses presented here indicates that the last common ancestor of all arthropods had a single RD gene, which has been independently duplicated in the chelicerate and crustacean/ insect lineages. All arthropod RD genes possess a runt DNA binding domain and a Groucho binding domain, suggesting that these proteins are able to act as transcriptional regulators, and both runt and lozenge have been shown to modulate developmental gene expression in Drosophila [20]. The functions of CG34145 and CG42267 are unknown, although CG42267 was identified as having a role in the control of cell survival in an RNAi screen
[61] and it has been suggested that cross-talk between RD proteins and the cell cycle may modulate cell cycle progression [62]; this would also be consistent with the ubiquitous expression of CG42267 and CG34145 during embryogenesis in Drosophila and A. mellifera [62]. Interestingly, one of the two sea urchin RD genes is also highly expressed in proliferating cells during embryogenesis [10], perhaps supporting an ancestral role for the RD genes in cell-cycle modulation.

The phylogenetic data indicates that runt is the ancestral RD gene in insects and crustaceans. In insects runt has a key role in segmentation [42], and orthologs of runt have been shown to be involved in the segmentation of two chelicerates $[46,47]$, raising the possibility that one of the functions of the single RD gene in the last common ancestor of all arthropods was a role in segmentation, and that the duplication and diversification of the RD genes has served to recruit the transcriptional co-repressor Groucho into new developmental niches, such as eye development [21].

\section{Conclusion}

$\mathrm{RD}$ genes are present in the genomes of organisms throughout metazoa, often found in multiple copies. Phylogenetic evidence supports the notion that these duplication events leading to multiple genomic copies of $\mathrm{RD}$ genes have occurred independently in the deuterostome and ecdysozoan lineages. Within arthropods, RD genes are also clustered, however phylogenetic data supports the independent duplication of RD genes in the chelicerate and crustacean/insect lineages. The RD genes in insects bear all the hallmarks of a functional gene cluster, in particular the very tight association of three of the four RD genes; CG42267, CG34145 and runt. However, in situ hybridisation of RNA expression did not suggest any temporal or spatial co-regulation of these RD genes in either Drosophila, which has a semi-dispersed cluster formation or in A. mellifera, which has an intact complex. It is possible that the retention of such a complex is not associated with functional constraints but is merely an artefact of tandem gene duplication; however, given the accelerated genome evolution observed in both Drosophila and mosquito species [63], but not T. castaneum [53], this would seem unlikely. While the significance of this RD gene cluster in insect species remains unknown the persistence of this cluster across all insect species implies functional importance.

\section{Methods \\ Beekeeping}

Apis mellifera were cultured using standard techniques in Dunedin, New Zealand. A. mellifera embryos were collected from frames removed from nucleus boxes containing small A. mellifera colonies. 


\section{Phylogenetic analyses and cluster identification}

Full-length RD metazoan protein sequences were obtained from relevant genome databases [25-28,30-34], or from NCBI. Unannotated RD proteins were predicted with the assistance of Augustus [37], and GeneScan [39]. Where required, runt domains were extracted from full length protein sequences using the hmmer suite of programs [64], against the Pfam runt hmm model (PF00853, 138 amino acids) [65].

Multiple alignments were carried out using ClustalX [66]. The multiple alignments were analysed using MrBAYES v3.1.2 [67] under the Jones model with default priors. The Jones model was chosen as the most appropriate model of amino acid substitution after preliminary analyses using MrBAYES with mixed models. The Monte Carlo Markov Chain search was run with four chains over 1000000 generations with trees sampled every 1000 generations. The first 250000 trees were discarded as 'burn-in'.

\section{Identification and cloning of RD genes}

Drosophila RD genes were identified in FlyBase [68], and A. mellifera orthologues of these Drosophila genes were identified by tBlastN [69] searches of the A. mellifera genome [26].

RNA was extracted from A. mellifera embryos using the RNeasy Mini Kit (Qiagen). cDNA was generated using Superscript II reverse transcriptase (Invitrogen) and an oligo-dT primer. The cDNA was used as a template for amplification of putative RD genes using the following oligonucleotide primers:

CG34145R ATGTGATCCATGACGCTCTG

CG34145F ATGTGCACTCCAGCCAGAAT

CG15455F AACAGCAGCAGCAACATCAG

CG15455R ATGTGGAGATCCCGTCTTGA

GB19482l GAGCAATTCATGGGGATACG

GB19482r ACTGGTTCCCGTACAACTGG

GB16431l CAAAACGAGGCAGACTCACA

GB 16431r GTGTCCCGACGGAAGAACAGT

GB 15836f AAGCGGTAGAGGAAAGAGTT

GB 15836r GGTGAAGACCTTGAAAGTGA

GB 11654 ATGCACTTACCGGAGGGCCCACTA
GB 11654 CTCGTGCTCGAGTCGCCCTAGTAG

runl CCACGACGAGTGTGATTAC

runr GACGACGCGTCCAAATA

lz3 TGATTCTGATTGACCGTGGA

lz5 CATGGGCATGAATCACTACG

Amplified DNA fragments were purified using the High Pure PCR purification kit (Roche) and cloned into pCRIITOPO (Invitrogen). The sequence and orientation of each cloned gene fragment was confirmed by DNA sequencing.

\section{In situ hybridisation}

A. mellifera embryos were collected and fixed as described [70]. Antisense or sense digoxigenin (DIG) labelled RNA probes were produced by in vitro transcription from linearised DNA templates containing cDNA fragments. In situ hybridisation on A. mellifera embryos were performed as previously described [70].

\section{Authors' contributions}

EJD and PKD performed the bioinformatics and phylogenetic analyses. MJW performed the A. mellifera ISH experiments. PKD performed the Drosophila ISH experiments and conceived the study. EJD, JMS, MJW and PKD wrote the manuscript.

\section{Additional material}

\section{Additional file 1}

multiple sequence alignment of full-length Drosophila RD proteins. ClustalX alignment of full-length RD protein sequences from 12 Drosophila species: D. melanogaster, D. simulans, D. sechellia, D. yakuba, D. erecta, D. ananassae, D. pseudoobscura, D. persimilis, $\mathrm{D}$. wilistoni, D. mojavensis, D. virilis, and $\mathrm{D}$. grimshawi.

Click here for file

[http://www.biomedcentral.com/content/supplementary/1471-

2164-9-558-S1.pdf]

\section{Additional file 2}

Phylogeny and multiple sequence alignment of endopterygotan insects full-length RD protein sequences. ClustalX alignment and Bayesian phylogeny of full-length RD protein sequences from a six endopterygotan insects: Drosophila melanogaster, Aedes. aegypti, Tribolium castaneum, Nasonia vitripennis, Apis mellifera and Bombyx mori. Alignment includes the outgroup Strongylocentrotus purpuratus.

Click here for file

[http://www.biomedcentral.com/content/supplementary/14712164-9-558-S2.pdf] 


\section{Additional file 3}

Multiple sequence alignment of full-length $R D$ protein sequences from exopterygotan and endopterygotan insects. ClustalX alignment of fulllength RD protein sequences from six insect species: Drosophila melanogaster, Tribolium castaneum, Acyrthosiphon pisum, Pediculus humanus, Bombyx mori and Apis mellifera. Alignment includes the outgroup Strongylocentrotus purpuratus.

Click here for file

[http://www.biomedcentral.com/content/supplementary/14712164-9-558-S3.pdf]

\section{Additional file 4}

Multiple sequence alignment of the RD protein sequences from a number of metazoan species. ClustalX alignment of $R D$ protein sequences from a number of metazoan species including representative species from the insects, crustaceans, chelicerates, cnidarians, lophotrochozoans, echinoderms and chordates.

Click here for file

[http://www.biomedcentral.com/content/supplementary/14712164-9-558-S4.pdf]

\section{Additional file 5}

Multiple sequence alignment of arthropod $R D$ domain sequences. ClustalX alignment of the RD from a number of arthropod species, including three insect species and four non-insect arthropods.

Click here for file

[http://www.biomedcentral.com/content/supplementary/14712164-9-558-S5.pdf]

\section{Acknowledgements}

The authors acknowledge the contribution of JR Alcock for discussions about portions of this data. The authors wish to acknowledge support from, and useful discussions with, the staff and students of the Laboratory for Evolution and Development. This work was funded by a RSNZ Marsden Grant (UOO40I) to PKD.

\section{References}

I. Wheeler JC, Shigesada K, Gergen JP, Ito K: Mechanisms of transcriptional regulation by Runt domain proteins. Semin Cell Dev Biol 2000, I I(5):369-375.

2. Ito Y: Oncogenic potential of the RUNX gene family: 'overview'. Oncogene 2004, 23(24):4198-4208.

3. Meyers S, Downing JR, Hiebert SW: Identification of AML-I and the $(8 ; 2 I)$ translocation protein (AML-I/ETO) as sequencespecific DNA-binding proteins: the runt homology domain is required for DNA binding and protein-protein interactions. Mol Cell Biol 1993, I3(I0):6336-6345.

4. Crute BE, Lewis AF, Wu Z, Bushweller JH, Speck NA: Biochemical and biophysical properties of the core-binding factor alpha2 (AMLI) DNA-binding domain. I Biol Chem 1996, 27I(42):2625I-26260.

5. Aronson BD, Fisher AL, Blechman K, Caudy M, Gergen JP: Grouchodependent and -independent repression activities of Runt domain proteins. Mol Cell Biol I997, I 7(9):558I-5587.

6. Rennert J, Coffman JA, Mushegian AR, Robertson AJ: The evolution of Runx genes I. A comparative study of sequences from phylogenetically diverse model organisms. BMC Evol Biol 2003, 3:

7. Sullivan JC, Sher D, Eisenstein M, Shigesada K, Reitzel AM, Marlow H, Levanon D, Groner Y, Finnerty JR, Gat U: The evolutionary origin of the Runx/CBF-beta transcription factors - studies of the most basal metazoans. BMC Evol Biol 2008, 8(I):228.

8. Coffman JA: Runx transcription factors and the developmental balance between cell proliferation and differentiation. Cell Biol Int 2003, 27(4):315-324.
9. Nam S, Jin YH, Li QL, Lee KY, Jeong GB, Ito Y, Lee J, Bae SC: Expression pattern, regulation, and biological role of runt domain transcription factor, run, in Caenorhabditis elegans. Mol Cell Biol 2002, 22(2):547-554.

10. Robertson AJ, Dickey CE, McCarthy JJ, Coffman JA: The expression of SpRunt during sea urchin embryogenesis. Mech Dev 2002, I I 7(I-2):327-330.

II. Gergen JP, Wieschaus EF: The Localized Requirements for a Gene Affecting Segmentation in Drosophila - Analysis of Larvae Mosaic for Runt. Dev Biol 1985, I 09(2):32I-335.

12. Nussleinvolhard C, Wieschaus E: Mutations Affecting Segment Number and Polarity in Drosophila. Nature 1980, 287(5785):795-80I.

13. Duffy JB, Gergen JP: The Drosophila segmentation gene runt acts as a position-specific numerator element necessary for the uniform expression of the sex-determining gene Sexlethal. Genes Dev 199I, 5(I2A):2176-2I87.

14. Torres M, Sanchez L: The Segmentation Gene Runt Is Needed to Activate Sex-Lethal, a Gene That Controls Sex Determination and Dosage Compensation in Drosophila. Genet Res 1992, 59(3): 189-198.

15. Dormand EL, Brand AH: Runt determines cell fates in the Drosophila embryonic CNS. Development 1998, I 25(9): I659-1667.

16. Duffy JB, Kania MA, Gergen JP: Expression and function of the Drosophila gene runt in early stages of neural development. Development |99|, I |3(4): |223-I230

17. Klingler M, Gergen JP: Regulation of runt transcription by Drosophila segmentation genes. Mech Dev 1993, 43(I):3-19.

18. Wolff C, Pepling M, Gergen P, Klingler M: Structure and evolution of a pair-rule interaction element: runt regulatory sequences in D. melanogaster and D. virilis. Mech Dev 1999, 80(I):87-99.

19. Tsai C, Gergen JP: Gap gene properties of the pair-rule gene runt during Drosophila segmentation. Development 1994, I 20(6): | 67|-|683.

20. Canon J, Banerjee U: Runt and Lozenge function in Drosophila development. Semin Cell Dev Biol 2000, I I (5):327-336.

21. Crew JR, Batterham P, Pollock JA: Developing compound eye in lozenge mutants of Drosophila : Lozenge expression in the R7 equivalence group. Dev Genes Evol 1997, 206(8):48I-493.

22. Flores GV, Daga A, Kalhor HR, Banerjee U: Lozenge is expressed in pluripotent precursor cells and patterns multiple cell types in the Drosophila eye through the control of cell-specific transcription factors. Development 1998 I 25( I8):368I-3687.

23. Nusse R: An ancient cluster of Wnt paralogues. Trends Genet 200I, I7(8):443

24. Mazet F, Amemiya CT, Shimeld SM: An ancient Fox gene cluster in bilaterian animals. Curr Biol 2006, I6(9):R3|4-316.

25. Clark AG, Eisen MB, Smith DR, Bergman CM, Oliver B, Markow TA, Kaufman TC, Kellis M, Gelbart W, lyer VN, et al: Evolution of genes and genomes on the Drosophila phylogeny. Nature 2007, 450(7167):203-218.

26. Honeybee Genome Sequencing Consortium: Insights into social insects from the genome of the honeybee Apis mellifera. Nature 2006, 443(7 I I 4):93I-949.

27. Nasonia Genome Project [http://www.hgsc.bcm.tmc.edu/ projects/nasonia/]

28. BeetleBase Tribolium Genome Database [http://www.bioin formatics.ksu.edu/BeetleBase]

29. Wang J, Xia Q, He X, Dai M, Ruan J, Chen J, Yu G, Yuan H, Hu Y, Li R, et al.: SilkDB: a knowledgebase for silkworm biology and genomics. Nucleic Acids Res 2005:D399-402.

30. Nene V, Wortman JR, Lawson D, Haas B, Kodira C, Tu ZJ, Loftus B, Xi Z, Megy K, Grabherr M, et al:: Genome sequence of Aedes aegypti, a major arbovirus vector. Science 2007 . 316(5832): 1718-1723.

31. Pea Aphid Genome Project [http://www.hgsc.bcm.tmc.edu/ projects/aphid/]

32. VectorBase, Pediculus humanus annotation, PhumUI.I [http://www.vectorbase.org]

33. wFleaBase Daphnia Water Flea Genome Database [http:// wfleabase.org/]

34. VectorBase, Ixodes scapularis annotation, IscaW I [http:// www.vectorbase.org] 
35. Tamura K, Subramanian S, Kumar S: Temporal patterns of fruit fly (Drosophila) evolution revealed by mutation clocks. Mol Biol Evol 2004, 21 (I):36-44.

36. Bhutkar A, Gelbart WM, Smith TF: Inferring genome-scale rearrangement phylogeny and ancestral gene order: a Drosophila case study. Genome Biol 2007, 8(I I):R236.

37. Stanke M, Morgenstern B: AUGUSTUS: a web server for gene prediction in eukaryotes that allows user-defined constraints. Nucleic Acids Res 2005:W465-467.

38. Holt RA, Subramanian GM, Halpern A, Sutton GG, Charlab R, Nusskern DR, Wincker P, Clark AG, Ribeiro JMC, Wides R, et al:: The genome sequence of the malaria mosquito Anopheles gambiae. Science 2002, 298(559I): 129-|49.

39. Yeh RF, Lim LP, Burge CB: Computational inference of homologous gene structures in the human genome. Genome Res $200 \mathrm{I}$, II (5):803-8I6.

40. Savard J, Tautz D, Richards S, Weinstock GM, Gibbs RA, Werren JH, Tettelin H, Lercher MJ: Phylogenomic analysis reveals bees and wasps (Hymenoptera) at the base of the radiation of Holometabolous insects. Genome Res 2006, I6(I I): I334-I338.

41. Krumlauf R: Hox genes in vertebrate development. Cell I994, 78(2): $|9|-20 \mid$.

42. Gergen JP, Butler BA: Isolation of the Drosophila Segmentation Gene Runt and Analysis of Its Expression during Embryogenesis. Genes Dev 1988, 2(9): I I79-1 I 93.

43. Osborne PW, Dearden PK: Expression of Pax group III genes in the honeybee (Apis mellifera). Dev Genes Evol 2005, 2I 5( 1 0):499-508.

44. Bataille L, Auge B, Ferjoux G, Haenlin M, Waltzer L: Resolving embryonic blood cell fate choice in Drosophila : interplay of GCM and RUNX factors. Development 2005, I 32(20):4635-4644.

45. Tepass U, Fessler LI, Aziz A, Hartenstein V: Embryonic origin of hemocytes and their relationship to cell death in Drosophila. Development 1994, I20(7):1829-1837.

46. Damen WGM, Weller M, Tautz D: Expression patterns of hairy, even-skipped, and runt in the spider Cupiennius salei imply that these genes were segmentation genes in a basal arthropod. Proc Natl Acad Sci USA 2000, 97(9):45 I5-45। 9 .

47. Dearden PK, Donly C, Grbic M: Expression of pair-rule gene homologues in a chelicerate: early patterning of the twospotted spider mite Tetranychus urticae. Development 2002, I29(23):546I-5472.

48. Srivastava M, Begovic E, Chapman J, Putnam NH, Hellsten U, Kawashima T, Kuo A, Mitros T, Salamov A, Carpenter ML, et al.: The Trichoplax genome and the nature of placozoans. Nature 2008, 454(7207):955-960.

49. Putnam NH, Srivastava M, Hellsten U, Dirks B, Chapman J, Salamov A, Terry A, Shapiro H, Lindquist E, Kapitonov VV, et al:: Sea anemone genome reveals ancestral eumetazoan gene repertoire and genomic organization. Science 2007, 3I7(5834):86-94.

50. Tijet N, Helvig C, Feyereisen R: The cytochrome P450 gene superfamily in Drosophila melanogaster: Annotation, intronexon organization and phylogeny. Gene 2001, 262:199-198.

5I. Engstrom PG, Ho Sui S], Drivenes O, Becker TS, Lenhard B: Genomic regulatory blocks underlie extensive microsynteny conservation in insects. Genome Res 2007, I7(12): 1898-1908.

52. Ferrier DE, Minguillon C: Evolution of the Hox/ParaHox gene clusters. Int I Dev Biol 2003, 47(7-8):605-6II.

53. Richards S, Gibbs RA, Weinstock GM, Brown SJ, Denell R, Beeman RW, Gibbs R, Bucher G, Friedrich M, Grimmelikhuijzen CJ, et al.: The genome of the model beetle and pest Tribolium castaneum. Nature 2008, 452(7 1 90):949-955.

54. Zdobnov EM, Bork P: Quantification of insect genome divergence. Trends Genet 2007, 23(I): 16-20.

55. Vieira CP, Vieira J, Hartl DL: The evolution of small gene clusters: Evidence for an independent origin of the maltase gene cluster in Drosophila virilis and Drosophila melanogaster. Mol Biol Evol 1997, I 4(10):985-993.

56. Ponce R, Hartl DL: The evolution of the novel Sdic gene cluster in Drosophila melanogaster. Gene 2006, 376(2): 174-183.

57. Klingler M, Soong J, Butler B, Gergen JP: Disperse versus compact elements for the regulation of runt stripes in Drosophila. Dev Biol 1996, I 77(I):73-84.

58. Liao D: Concerted evolution: molecular mechanism and biological implications. Am J Hum Genet 1999, 64(I):24-30.
59. Peel AD, Telford MJ, Akam M: The evolution of hexapod engrailed-family genes: evidence for conservation and concerted evolution. Proc Biol Sci 2006, 273(1595): 1733- 1742.

60. Fan C, Chen Y, Long M: Recurrent tandem gene duplication gave rise to functionally divergent genes in Drosophila. Mol Biol Evol 2008, 25(7): | $45 \mid-1458$.

6I. Boutros M, Kiger AA, Armknecht S, Kerr K, Hild M, Koch B, Haas SA Paro R, Perrimon N: Genome-wide RNAi analysis of growth and viability in Drosophila cells. Science 2004, 303(5659):832-835.

62. Nimmo R, Woollard A: Worming out the biology of Runx. Dev Biol 2008, 313(2):492-500.

63. Savard J, Tautz D, Lercher MJ: Genome-wide acceleration of protein evolution in flies (Diptera). BMC Evol Biol 2006, 6:7.

64. Eddy SR: Profile hidden Markov models. Bioinformatics 1998 I 4(9):755-763

65. Finn RD, Tate J, Mistry J, Coggill PC, Sammut SJ, Hotz HR, Ceric G, Forslund K, Eddy SR, Sonnhammer EL, et al:: The Pfam protein families database. Nucleic Acids Res 2008:D28I-288.

66. Thompson JD, Higgins DG, Gibson TJ: Clustal-W - Improving the Sensitivity of Progressive Multiple Sequence Alignment through Sequence Weighting, Position-Specific Gap Penalties and Weight Matrix Choice. Nucleic Acids Res 1994 22(22):4673-4680

67. Ronquist F, Huelsenbeck JP: MrBayes 3: Bayesian phylogenetic inference under mixed models. Bioinformatics 2003, 19(12): 1572-1574.

68. Drysdale R: FlyBase : a database for the Drosophila research community. Methods Mol Biol 2008, 420:45-59.

69. Altschul SF, Gish W, Miller W, Myers EW, Lipman DJ: Basic Local Alignment Search Tool. J Mol Biol 1990, 215(3):403-410.

70. Osborne P, Dearden PK: Non-radioactive in-situ hybridisation to honeybee embryos and ovaries. Apidologie 2005, 36(I): $113-118$.

Publish with Biomed Central and every scientist can read your work free of charge

"BioMed Central will be the most significant development for disseminating the results of biomedical research in our lifetime. "

Sir Paul Nurse, Cancer Research UK

Your research papers will be:

- available free of charge to the entire biomedical community

- peer reviewed and published immediately upon acceptance

- cited in PubMed and archived on PubMed Centra

- yours - you keep the copyright
BioMedcentral 